\title{
Higher Serum Uric Acid is a Risk Factor of Vertebral Fractures in Postmenopausal Women with Type 2 Diabetes Mellitus
}

\author{
Authors \\ Ken-ichiro Tanaka, Ippei Kanazawa, Masakazu Notsu, Toshitsugu Sugimoto
}

\author{
Affiliation \\ Department of Internal Medicine 1, Shimane University \\ Faculty of Medicine, Japan
}

\section{Keywords}

uric acid, hyperuricemia, vertebral fracture, bone,

diabetes mellitus

$\begin{array}{ll}\text { received } & 03.09 .2018 \\ \text { revised } & 23.11 .2018 \\ \text { accepted } & 04.12 .2018\end{array}$

Bibliography

DOI https://doi.org/10.1055/a-0815-4954

Published online: 18.12.2018

Exp Clin Endocrinol Diabetes 2020; 128: 66-71

(c) J. A. Barth Verlag in Georg Thieme Verlag KG Stuttgart . New York

ISSN 0947-7349

\section{Corresponding}

Ippei Kanazawa, MD PhD

Department of Internal Medicine 1

Shimane University Faculty of Medicine

Izumo 693-8501

Japan

Tel $+81 / 853 / 202183$ ext. 2183 , Fax: $+81 / 853 / 238650$

ippei.k@med.shimane-u.ac.jp

\section{ABSTRACT}

Purpose Serum uric acid (UA) level may affect bone metabolism because it has an anti-oxidative effect. However, whether serum UA level is associated with a fracture risk in type 2 diabetes mellitus (T2DM) is unclear. We thus aimed to clarify the association between serum UA and bone parameters in T2DM. Methods We conducted a cross-sectional study to investigate the association of serum UA with bone mineral density (BMD) at lumbar spine (LS) and femoral neck (FN), bone turnover markers such as osteocalcin and urine type I collagen crosslinked N-telopeptide (uNTX), and the prevalence of vertebral fractures (VF) in 356 postmenopausal women and 512 men with T2DM.

Results Multiple regression analyses adjusted for age, duration of diabetes, hemoglobin A1c, body mass index and log (serum creatinine) showed that serum UA level was significantly and negatively associated with uNTX in postmenopausal women with T2DM, whereas it was not associated with osteocalcin or BMD at each site. In men, serum UA was not associated with BMD or bone turnover markers. Because postmenopausal women with VF were significantly older and had longer duration of diabetes, higher serum creatinine level and lower BMD than those without it, logistic regression analyses adjusted for these confounding factors were performed. Higher serum UA level was significantly associated with the presence of VF.

Conclusions The present study showed that higher serum UA is a risk factor for VF independently of BMD in postmenopausal women with T2DM.

$\begin{array}{ll}\text { ABBREVIATIONS } \\ \text { T2DM } & \text { Type } 2 \text { diabetes mellitus } \\ \text { HbA1 } & \text { chemoglobin A1c } \\ \text { LS } & \text { lumbar spine } \\ \text { BMD } & \text { bone mineral density } \\ \text { FN } & \text { femoral neck } \\ \text { NTX } & \text { type I collagen cross-linked N-telopeptide } \\ \text { BMI } & \text { body mass index } \\ \text { VF } & \text { vertebral fracture } \\ \text { DXA } & \text { dual-energy } x \text {-ray absorptiometry }\end{array}$

\section{Introduction}

The number of patients with type 2 diabetes mellitus (T2DM) is rapidly increasing especially among elderly people, and it is estimated that 439 million people will have T2DM by 2030 [1]. Their quality of life (QOL) is decreased by various complications, such as diabetic neuropathy, retinopathy and nephropathy as well as cardiovascular diseases. It has been shown that patients with T2DM have a higher fracture risk compared to non-diabetics [2, 3]; thus, osteoporosis is now recognized as a complication of T2DM. Moreover, we have shown that lower bone mineral density (BMD) and the presence of vertebral fracture (VF) increase the risk of all-cause mortality in patients with T2DM [4]. Therefore, it is an urgent task to clarify which risk factors are involved in the risk of fracture in patients with T2DM. 
Hyperuricemia is defined as an abnormally high level of serum uric acid (UA) and it is often accompanied by metabolic syndrome and T2DM. Previous studies have shown that UA acts as an antioxidant in vivo [5]. However, the serum UA level is needed to be controlled within the proper range by diet and anti-hyperuricemic drugs, because hyperuricemia causes gout, cardiovascular disorders, hypertension and a progressive renal disease [6-8]. Previous studies reported that serum UA level might be associated with BMD and the fracture risk [9-14]. Nabipour et al. showed that serum UA level was positively associated with BMD and negatively with bone resorption markers in elder men, and that those with high serum UA level had lower prevalence of osteoporotic fractures [9]. Makovey et al. reported that serum UA level was positively associated with BMD in women, and that those with high serum UA level had slower reduction in BMD [10]. In contrast, Mehta et al. showed that men with high UA level had an increased risk of hip fracture compared to those with low UA level [14]. Thus, the effect of serum UA on BMD and the fracture risk still remains unclear. These controversial results may be caused by the difference in background characteristics of subjects.

The pathophysiology of bone fragility in T2DM is considered to be different from that of primary osteoporosis, because patients with T2DM have increased fracture risk despite normal or increased BMD [15]. Previous studies suggested that oxidative stress may be involved in diabetes-induced bone fragility [16]. Therefore, the roles of UA in bone metabolism should be investigated in diabetesinduced bone fragility. A couple of cross-sectional studies showed that serum UA levels were positively associated with BMD in patients with T2DM $[23,24]$. However, Yan et al. reported that serum UA was not associated with the presence of clinical fracture although the association was significant before adjusting for BMD [23]. Therefore, it is still unclear whether or not serum UA is involved in fragility fracture risk in patients with T2DM. We thus conducted a cross-sectional study to clarify this issue in postmenopausal women and men aged 50 years and older with T2DM.

\section{Methods}

\section{Subjects}

This is a cross-sectional study examining the association between serum UA level and fracture risk in patients with T2DM. Patients admitted to Shimane University Hospital for evaluation and treatment for T2DM except for having malignant diseases, infection, necessity of operation and transplantation, and other special purposes from 2002 - 2014 were screened. In our hospital, osteoporosis has been checked as a diabetic complication. According to the hospital records, 517 women and 814 men were admitted. Among them, serum UA level and bone parameters such as bone turnover markers, BMD, and the presence of VF were examined in 379 postmenopausal women and 713 men with T2DM. We then excluded 1) premenopausal women who had spontaneous menses within 1 year, 2) men younger than 50 years, 3) patients who had renal dysfunction [estimated glomerular filtration rate (eGFR) $<30 \mathrm{~mL}$ / $\mathrm{min} / 1.73 \mathrm{~m}^{2}$ ], 4) patients had taken drugs for hyperuricemia and osteoporosis such as bisphosphonate and hormone replacement therapy, as well as thiazolidinedione and glucocorticoid which are known to affect bone metabolism. Finally, 356 postmenopausal women and 512 men aged 50 years and older with T2DM were included in this analysis. Patient characteristics of demographic and biochemical parameters, BMD and the prevalence of VF are shown in $>$ Table 1. This study was approved by the ethical review board of Shimane University Faculty of Medicine and complied with the Helsinki Declaration. The requirement for informed patient consent was waived because no intervention and further examinations were performed.

\section{Biochemical measurements}

On the second day after admission, blood and urine samples were collected after overnight fasting. Biochemical markers were measured by standard methods as previously described [17, 18]. Hemoglobin A1c (HbA1c) was determined with high-performance liquid chromatography. The value for $\mathrm{HbA} 1 \mathrm{c}$ was estimated as the $\mathrm{Na}$ tional Glycohemoglobin Standardization Program equivalent value calculated using the formula HbA1c (\%) = HbA1c (Japan Diabetes Society) (\%) $+0.4 \%[18]$. Total osteocalcin and urine type I collagen cross-linked N-telopeptide (uNTX) levels were measured by radioimmunoassay and enzyme-linked immunosorbent assay, respectively. The coefficients of variation (CV) of osteocalcin and uNTX measurements were 5.5 and $5.7 \%$, respectively.

\section{BMD measurements}

BMD of the lumbar spine at L2-4 (LS) and femoral neck (FN) was evaluated by dual-energy $x$-ray absorptiometry (QDR4500, Hologic Co., Waltham, MA, USA). The CV (precision) of measurements of LS- and FN-BMD by our methods were 0.9 and $1.7 \%$, respectively.

- Table 1 Baseline characteristics of subjects.

\begin{tabular}{|c|c|c|c|}
\hline & Women & Men & $\mathbf{p}$ \\
\hline Number of patients & 356 & 512 & \\
\hline Age (years) & $67.4 \pm 9.7$ & $65.7 \pm 8.8$ & 0.009 \\
\hline $\begin{array}{l}\text { Duration of diabetes } \\
\text { (years) }\end{array}$ & $12.2 \pm 10.4$ & $12.6 \pm 9.6$ & 0.672 \\
\hline Body height (cm) & $150.2 \pm 5.7$ & $163.8 \pm 6.3$ & $<0.001$ \\
\hline Body weight (kg) & $55.4 \pm 11.3$ & $62.0 \pm 10.4$ & $<0.001$ \\
\hline Body mass index $\left(\mathrm{kg} / \mathrm{m}^{2}\right)$ & $24.5 \pm 4.6$ & $23.1 \pm 3.4$ & $<0.001$ \\
\hline Serum creatinine (mg/dL) & $0.64 \pm 0.18$ & $0.81 \pm 0.23$ & $<0.001$ \\
\hline HbA1c (NGSP) (\%) & $8.8 \pm 2.3$ & $8.8 \pm 2.2$ & 0.796 \\
\hline Serum uric acid (mg/dL) & $4.6 \pm 1.3$ & $5.2 \pm 1.4$ & $<0.001$ \\
\hline LS BMD $\left(\mathrm{g} / \mathrm{cm}^{2}\right)$ & $0.88 \pm 0.18$ & $1.03 \pm 0.21$ & $<0.001$ \\
\hline LS T score & $-1.20 \pm 1.57$ & $-0.09 \pm 1.69$ & $<0.001$ \\
\hline LS Z score & $0.64 \pm 1.29$ & $0.50 \pm 1.18$ & 0.117 \\
\hline FN BMD $\left(\mathrm{g} / \mathrm{cm}^{2}\right)$ & $0.63 \pm 0.13$ & $0.74 \pm 0.12$ & $<0.001$ \\
\hline FN T score & $-1.48 \pm 1.14$ & $-0.96 \pm 0.98$ & $<0.001$ \\
\hline FN Z score & $0.37 \pm 1.19$ & $0.16 \pm 1.07$ & 0.017 \\
\hline Osteocalcin (ng/mL) & $6.7 \pm 2.9$ & $5.2 \pm 2.7$ & $<0.001$ \\
\hline uNTX (nMBCE/mM-Cr) & $54.7 \pm 33.8$ & $38.3 \pm 47.1$ & $<0.001$ \\
\hline Vertebral fracture n, (\%) & $109(30.6 \%)$ & $188(36.7 \%)$ & 0.054 \\
\hline \multicolumn{4}{|c|}{$\begin{array}{l}\text { Data are means } \pm \text { SD.; HbA1c, hemoglobin A1c; LS, lumbar spine } \\
\text { 2-4; BMD, bone mineral density; FN, femoral neck; uNTX, urinary } \\
\text { type I collagen cross-linked N-telopeptide. }\end{array}$} \\
\hline
\end{tabular}


T-scores indicate a deviation from the averaged BMD in sex-matched young Japanese normal reference mean, and Z-scores indicate a deviation from the averaged BMD in normal age- and sex-matched Japanese subjects in the standardized normal distribution.

\section{Assessment of VF}

Lateral radiographic films of the thoracic and lumbar spine were taken during the same week as the serum collection. The anterior, central and posterior heights of each of the 13 vertebral bodies from T4 to L4 were measured. A vertebral fracture was diagnosed if at least one of the 3 height measurements along the length of the same vertebrae had decreased by $>20 \%$ compared with the height of the nearest uncompressed vertebral body [19].

\section{Statistical analysis}

Data are expressed as mean \pm standard deviation (SD). Because serum creatinine showed a skewed distribution, it was transformed to logarithm before multiple regression and logistic analyses. All analyses were carried out using StatView (Abacus Concepts, Berkeley, CA, USA). A p value $<0.05$ was considered significant.

\section{Results}

\section{Association of serum UA with BMD and bone turnover markers}

Simple correlation analysis showed that the serum UA level was significantly and positively correlated with LS-BMD (women: $r=0.15$, $p=0.006$, men: $r=0.12, p=0.007)$ and negatively with $u N T X$ (women: $r=-0.25, p<0.001$, men: $r=-0.17, p<0.001$ ) in both women and men, whereas it was not correlated with FN-BMD or osteocalcin.

Next, we performed multiple regression analyses adjusted for age, duration of diabetes, $\mathrm{HbA} 1 \mathrm{c}$, body mass index (BMI) and log (serum creatinine) ( $\triangleright$ Table 2 ). The serum UA level was significantly and negatively associated with uNTX in women $(\beta=-0.22$, $p=0.003$ ), whereas it was not associated with BMD or osteocalcin. There was no association of the serum UA level with BMD or bone turnover markers in men.

\section{Association between serum UA and the presence of VF}

Next, we compared demographic, biochemical parameters and BMD between subjects with and without VF. As shown in $>$ Table 3 , women with VF were significantly older and had longer duration of diabetes and higher serum creatinine level than those without it $(p<0.001$ for all). BMD and T score at LS and FN were significantly lower in women with VF than those without it ( $p<0.001$ for all). The uNTX level was significantly higher in women with VF than those without it $(p=0.032)$. Men with VF were significantly older and had lower $\mathrm{HbA} 1 \mathrm{c}$ than those without it $(\mathrm{p}<0.001$ and $p=0.017$, respectively). BMD, T and Z scores at FN as well as T score at LS were significantly lower in men with VF than those without it (at least $\mathrm{p}<0.03)$.

Finally, we performed logistic regression analyses adjusted for age, duration of diabetes, log (serum creatinine), and BMD as well as UNTX because these parameters showed significant deviations between women with and without VF ( $\triangleright$ Table 3 ). As shown in
- Table 2 Multiple regression analyses between serum uric acid level and bone parameters.

\begin{tabular}{|l|l|l|l|l|}
\hline \multirow{2}{*}{} & \multicolumn{2}{|c|}{ Women } & \multicolumn{2}{c|}{ Men } \\
\cline { 2 - 5 } & $\boldsymbol{\beta}$ & $\mathbf{p}$ & $\boldsymbol{\beta}$ & $\mathbf{p}$ \\
\hline LS BMD & 0.12 & 0.071 & 0.07 & 0.166 \\
\hline FN BMD & 0.06 & 0.375 & -0.05 & 0.382 \\
\hline Osteocalcin & 0.02 & 0.806 & -0.07 & 0.204 \\
\hline UNTX & -0.22 & 0.003 & -0.08 & 0.145 \\
\hline
\end{tabular}

Multiple regression analyses adjusted for age, duration of diabetes, $\mathrm{HbA1c}$, body mass index and log (serum creatinine) were performed. LS, lumbar spine 2-4; BMD, bone mineral density; FN, femoral neck; uNTX, urinary type I collagen cross-linked $\mathrm{N}$-telopeptide.

- Table 4, higher serum UA level was significantly associated with the presence of VF in women [odds ratio $(O R)=1.38,95 \%$ confidence interval $(\mathrm{Cl})=1.01-1.88$ per SD increase, $\mathrm{P}=0.041$ adjusted for $L S-B M D$ and $O R=1.40,95 \% \mathrm{Cl}=1.00-1.96$ per SD increase, $\mathrm{p}=0.048$ adjusted for FN-BMD]. Moreover, the association was still significant after additional adjustment for uNTX (OR $=1.46,95 \%$ $\mathrm{Cl}=1.04-2.07$ per SD increase, $\mathrm{p}=0.031$ adjusted for $\mathrm{LS}-\mathrm{BMD}$ and $\mathrm{OR}=1.42,95 \% \mathrm{Cl}=1.00-2.02$ per SD increase, $\mathrm{p}=0.047$ adjusted for FN-BMD). In contrast, there was no association between the serum UA level and the presence of VF in men.

\section{Discussion}

Bone is constantly renewed by osteoblastic bone formation and osteoclastic bone resorption. Previous studies have shown that patients with T2DM have a low bone turnover [20,21], suggesting that bone remodeling is impaired and debased bone would be accumulated in patients with T2DM. In this study, we found that the serum UA level was negatively associated with uNTX in postmenopausal women with T2DM even after adjustment for various confounding factors. This finding indicates that the increased UA may induce further low bone remodeling in case of T2DM. Therefore, whether the higher serum UA level is beneficial or harmful should be examined in patients with T2DM. In this study, we found that the serum UA level was significantly and positively associated with the presence of VF in postmenopausal women with T2DM, suggesting that high serum UA level is a risk factor for VF in case of postmenopausal women with T2DM.

Previous studies showed that serum UA level was positively associated with BMD in patients with T2DM $[23,24]$. In this study, simple correlation analysis showed that serum UA level was positively correlated with LS-BMD and negatively with UNTX in both women and men, and multiple regression analysis showed that serum UA level was marginally and positively associated with LSBMD in women. These findings seem to be consistent with the previous ones. However, it has been shown that T2DM have BMD-independent risk of osteoporotic fractures [15, 22], indicating that impaired bone quality is involved in the risk of fracture rather than BMD reduction in patients with T2DM. Therefore, it remains unclear whether or not serum UA is involved in the fracture risk in T2DM. Yan et al. previously reported that serum UA was not associated with BMD-independent risk of self-reported clinical fractures 
- Table 3 Demographic and biochemical parameters of subjects with and without vertebral fracture.

\begin{tabular}{|c|c|c|c|c|c|c|}
\hline & \multicolumn{3}{|c|}{ Women } & \multicolumn{3}{|c|}{ Men } \\
\hline & with VF & without VF & $\mathbf{p}$ & with VF & without VF & $\mathbf{p}$ \\
\hline Number of patients & 109 & 239 & & 188 & 310 & \\
\hline Age (years) & $71.7 \pm 9.2$ & $65.4 \pm 9.2$ & $<0.001$ & $67.9 \pm 8.9$ & $64.5 \pm 8.4$ & $<0.001$ \\
\hline Duration of diabetes (years) & $15.5 \pm 10.3$ & $11.0 \pm 10.1$ & $<0.001$ & $13.7 \pm 9.7$ & $12.1 \pm 9.6$ & 0.108 \\
\hline Body mass index $\left(\mathrm{kg} / \mathrm{m}^{2}\right)$ & $24.4 \pm 5.2$ & $24.6 \pm 4.3$ & 0.755 & $23.0 \pm 3.3$ & $23.2 \pm 3.4$ & 0.679 \\
\hline Serum creatinine (mg/dL) & $0.69 \pm 0.23$ & $0.62 \pm 0.15$ & $<0.001$ & $0.81 \pm 0.24$ & $0.82 \pm 0.23$ & 0.804 \\
\hline HbA1c (NGSP) (\%) & $8.8 \pm 2.4$ & $8.9 \pm 2.3$ & 0.681 & $8.5 \pm 1.9$ & $9.0 \pm 2.3$ & 0.017 \\
\hline Serum uric acid (mg/dL) & $4.8 \pm 1.4$ & $4.5 \pm 1.2$ & 0.071 & $5.1 \pm 1.5$ & $5.3 \pm 1.4$ & 0.313 \\
\hline LS BMD $\left(\mathrm{g} / \mathrm{cm}^{2}\right)$ & $0.83 \pm 0.20$ & $0.90 \pm 0.16$ & $<0.001$ & $1.01 \pm 0.20$ & $1.05 \pm 0.22$ & 0.085 \\
\hline LS T score & $-1.65 \pm 1.76$ & $-1.00 \pm 1.44$ & $<0.001$ & $-0.31 \pm 1.64$ & $0.05 \pm 1.73$ & 0.026 \\
\hline LS Z score & $0.46 \pm 1.26$ & $0.72 \pm 1.31$ & 0.101 & $0.38 \pm 1.15$ & $0.58 \pm 1.21$ & 0.077 \\
\hline FN BMD $\left(\mathrm{g} / \mathrm{cm}^{2}\right)$ & $0.59 \pm 0.23$ & $0.65 \pm 0.12$ & $<0.001$ & $0.72 \pm 0.12$ & $0.76 \pm 0.13$ & 0.001 \\
\hline FN T score & $-1.83 \pm 1.16$ & $-1.29 \pm 1.08$ & $<0.001$ & $-1.17 \pm 0.92$ & $-0.83 \pm 1.00$ & $<0.001$ \\
\hline FN Z score & $0.17 \pm 1.27$ & $0.46 \pm 1.15$ & 0.060 & $0.00 \pm 1.00$ & $0.25 \pm 1.11$ & 0.019 \\
\hline Osteocalcin (ng/mL) & $6.69 \pm 3.48$ & $6.77 \pm 2.69$ & 0.844 & $5.14 \pm 2.88$ & $5.29 \pm 2.50$ & 0.578 \\
\hline uNTX (nMBCE/mM-Cr) & $60.3 \pm 42.2$ & $51.1 \pm 28.4$ & 0.032 & $37.5 \pm 20.7$ & $38.7 \pm 58.6$ & 0.790 \\
\hline
\end{tabular}

D Table 4 Association of serum uric acid level with the presence of vertebral fracture.

\begin{tabular}{|c|c|c|c|c|}
\hline & \multicolumn{2}{|c|}{ Women } & \multicolumn{2}{|c|}{ Men } \\
\hline & OR (95\% Cl) & p & OR (95\% Cl) & $\mathbf{p}$ \\
\hline Model 1 & $\begin{array}{l}1.38 \\
(1.01-1.88)\end{array}$ & 0.041 & $\begin{array}{l}1.05 \\
(0.84-1.32)\end{array}$ & 0.654 \\
\hline Model 2 & $\begin{array}{l}1.40 \\
(1.00-1.96)\end{array}$ & 0.048 & $\begin{array}{l}0.99 \\
(0.79-1.25)\end{array}$ & 0.940 \\
\hline Model 3 & $\begin{array}{l}1.46 \\
(1.04-2.07)\end{array}$ & 0.031 & $\begin{array}{l}1.05 \\
(0.82-1.33)\end{array}$ & 0.708 \\
\hline Model 4 & $\begin{array}{l}1.42 \\
(1.01-2.02)\end{array}$ & 0.047 & $\begin{array}{l}1.01 \\
(0.79-1.29)\end{array}$ & 0.924 \\
\hline
\end{tabular}

Logistic regression analyses were performed.; Model 1: adjusted for age, duration of diabetes, log (serum creatinine) and LS BMD.; Model 2: adjusted for age, duration of diabetes, $\log$ (serum creatinine) and FN BMD.; Model 3: adjusted for Model 1 and uNTX.; Model 4: adjusted for Model 2 and uNTX. OR, odds ratio; $\mathrm{Cl}$, confidence interval.

although serum UA was inversely associated with clinical fracture risk in Chinese population with T2DM [23]. In contrast, in the present study, the serum UA level was positively associated with the presence of VF in logistic regression analyses adjusted for LS-BMD and FN-BMD. It is known that VF is the most frequent osteoporotic fracture, and that most of VF occur without pain [32]. Thus, silent VF may not be reported as clinical fractures and should be diagnosed by lateral radiographic films of the thoracic and lumbar spine, as in this study. Moreover, previous studies have shown that duration of T2DM is associated with fracture risk. Majumdar et al. reported that patients with T2DM loner than 10 years are at particularly high risk of fracture [33]. Mean diabetic duration of the subjects in the previous study by Yan et al. was 7.41 years and 8.53 years in men and postmenopausal women, respectively [23], while that of our patients was 12.6 years and 12.2 years in men and post- menopausal women, respectively. Therefore, methodology to collect fragility fracture and fracture site as well as background such as duration of diabetes might be related to the contrasting findings between the previous study and ours.

Previous studies have shown that a functional urate transporter 1 (Urat 1 ) is expressed in various cells such as vascular endothelial and smooth muscle cells [25] as well as adipocytes [26]. Kang et al. reported that increased UA uptake into vascular cells caused local inflammation and reduced nitric oxide release even at physiologic concentration of UA ( 6 to $12 \mathrm{mg} / \mathrm{dL}$ ) [27]. Baldwin et al. showed that UA induced an increase in the production of monocyte chemotactic protein-1, an essential adipokine inducing the proinflammatory state in adipose tissue, and a decrease in the production of adiponectin, an anti-inflammatory adipokine [26]. Although there are no reports showing the expression of the urate transporter in bone, Ahn et al. demonstrated that UA suppressed the differentiation of osteoclast-like cells in vitro [28]. In this study, serum UA level was negatively associated with uNTX, which is consistent with the in vitro study [28]. However, to our knowledge, there are no studies examining the effects of UA on osteoblasts and osteocytes so far. Further studies are thus needed to clarify the association between UA and bone metabolism.

Interestingly, the significant association of serum UA with uNTX and VF was only seen in postmenopausal women with T2DM in this study although differences in background characteristics such as age, BMI and serum UA might contribute to the gender difference. Estradiol, the strong stimulator of bone growth, is known to be responsible for maintaining bone strength. It is known that increased oxidative stress enhances osteoclast activity and is involved in postmenopausal bone loss [29]. Serum UA may act as a protective factor against the oxidative stress-induced osteoclast activity through its antioxidative capacity especially in postmenopausal women. Indeed, several studies showed that serum UA level was negatively 
associated with bone resorption markers [28, 30], and we also confirmed the negative association between serum UA and UNTX in postmenopausal women with T2DM. The suppressive effects of UA on bone resorption might be beneficial for postmenopausal osteoporosis although whether or not it is also beneficial for diabetesinduced bone fragility is necessary to be clarified. In contrast, it has been shown that estradiol suppresses the expression of Urat1, which resorbs UA on the luminal side of the tubular epithelium, in the mouse kidney [31]. Thus, sudden loss of estradiol after menopause and the following rapid rise in serum UA might be associated with the negative association between UA and VF. To clarify the role of estradiol in the association between UA and fracture risk, we need to investigate and compare the association between preand postmenopausal women in future.

There are several limitations in our study. First, the sample size was not large enough to make definite conclusions. Second, we analyzed only subjects who visited our hospital, a tertiary center, for treatment of diabetes mellitus. Therefore, the participants enrolled in this study might have relatively severe states of the disorders and might not be representative of Japanese patients. Third, non-diabetic control subjects were not examined in this study. Therefore, we can't compare the contribution of serum UA to bone metabolism and fracture risk between T2DM and non-diabetics. Forth, we need to examine inflammation markers and reactive oxygen species to identify the underlying mechanism of the relation between $U A$ and VF risk. Finally, we need to examine not only cross-sectional studies but also longitudinal ones to understand the causal relationship between serum UA level and VF in T2DM.

In conclusion, this study showed that high serum UA level is a risk factor for VF independently of BMD in postmenopausal women with T2DM. These findings might give a clue for comprehensive prevention and treatment of osteoporosis in patients with T2DM and hyperuricemia.

\section{Acknowledgments}

This study was partly supported by research grant from Eli Lilly Japan.

\section{Conflict of Interest}

No conflict of interest has been declared by the authors.

\section{References}

[1] Shaw JE, Sicree RA, Zimmet PZ. Global estimates of the prevalence of diabetes for 2010 and 2030. Diabetes Res Clin Pract 2010; 87: 4-14

[2] Janghorbani M, Van Dam RM, Willett WC, Hu FB. Systematic review of type 1 and type 2 diabetes mellitus and risk of fracture. Am J Epidemiol 2007; 166: 495-505

[3] Fan Y, Wei F, Lang Y, Liu Y. Diabetes mellitus and risk of hip fractures: A meta-analysis. Osteoporos Int 2016; 27: 219-228
[4] Miyake H, Kanazawa I, Sugimoto T. Association of Bone Mineral Density, Bone Turnover Markers, and Vertebral Fractures with All-Cause Mortality in Type 2 Diabetes Mellitus. Calcif Tissue Int 2018; 102: $1-13$

[5] Glantzounis GK, Tsimoyiannis EC, Kappas AM, Galaris DA. Uric acid and oxidative stress. Curr Pharm Des 2005; 11: 4145-4151

[6] Lippi G, Montagnana M, Franchini M et al. The paradoxical relationship between serum uric acid and cardiovascular disease. Clin Chim Acta 2008; 392: 1-7

[7] Verdecchia P, Schillaci G, Reboldi G et al. Relation between serum uric acid and risk of cardiovascular disease in essential hypertension. The PIUMA study. Hypertension 2000; 36: 1072-1078

[8] Johnson R], Kang DH, Feig D et al. Is there a pathogenetic role for uric acid in hypertension and cardiovascular and renal disease? Hypertension 2003; 41: 1183-1190

[9] Nabipour I, Sambrook PN, Blyth FM et al. Serum uric acid is associated with bone health in older men: A cross-sectional population-based study. J Bone Miner Res 2011; 26: 955-964

[10] Makovey J, Macara M, Chen JS et al. Serum uric acid plays a protective role for bone loss in peri- and postmenopausal women: A longitudinal study. Bone 2013; 52: 400-406

[11] Ishii S, Miyao M, Mizuno $Y$ et al. Association between serum uric acid and lumbar spine bone mineral density in peri- and postmenopausal Japanese women. Osteoporos Int 2014; 25: 1099-1105

[12] Lane NE, Parimi N, Lui LY et al. Osteoporotic Fractures in Men Study Group. Association of serum uric acid and incident nonspine fractures in elderly men: The Osteoporotic Fractures in Men (MrOS) study. J Bone Miner Res 2014; 29: 1701-1707

[13] Zhang D, Bobulescu IA, Maalouf NM et al. Relationship between serum uric Acid and bone mineral density in the general population and in rats with experimental hyperuricemia. J Bone Miner Res 2015; 30: 992-999

[14] Mehta T, Bůžková P, Sarnak MJ et al. Serum urate levels and the risk of hip fractures: Data from the Cardiovascular Health Study. Metabolism 2015; 64: 438-446

[15] Vestergaard P. Discrepancies in bone mineral density and fracture risk in patients with type 1 and type 2 diabetes -A meta-analysis. Osteoporos Int 2007; 18: 427-444

[16] Napoli N, Chandran M, Pierroz DD et al. IOF Bone and Diabetes Working Group. Mechanisms of diabetes mellitus-induced bone fragility. Nat Rev Endocrinol 2017; 13: 208-219

[17] Kanazawa I, Yamaguchi T, Sugimoto T. Serum insulin-like growth factor-l is amarker for assessing the severity of vertebral fractures in postmenopausal women with type 2 diabetes mellitus. Osteoporos Int 2011; 22: 1191-1198

[18] Kanazawa I, Yamamoto M, Yamaguchi T, Sugimoto T. Effects of metformin and pioglitazone on serum pentosidine levels in type 2 diabetes mellitus. Exp Clin Endocrinol Diabetes 2011; 119: 362-365

[19] Genant HK, Jergas M, Palermo L et al. Comparison of semiquantitative visual and quantitative morphometric assessment of prevalent and incident vertebral fractures in osteoporosis The Study of Osteoporotic Fractures Research Group. J Bone Miner Res 1996; 11: 984-996

[20] Hygum K, Starup-Linde J, Harsløf T et al. Mechanisms in Endocrinology: Diabetes mellitus, a state of low bone turnover - A systematic review and meta-analysis. Eur J Endocrinol 2017; 176: R137-R157

[21] Kunutsor SK, Apekey TA, Laukkanen JA. Association of serum total osteocalcin with type 2 diabetes and intermediate metabolic phenotypes: Systematic review and meta-analysis of observational evidence. Eur J Epidemiol 2015; 30: 599-614 
[22] Yamamoto M, Yamaguchi T, Yamauchi M, Kaji H, Sugimoto T. Diabetic patients have an increased risk of vertebral fractures independent of bone mineral density or diabetic complications. J Bone Miner Res 2009; 24: 702-709

[23] Yan P, Zhang Z, Wan Q et al. Association of serum uric acid with bone mineral density and clinical fractures in Chinese type 2 diabetes mellitus patients: A cross-sectional study. Clin Chim Acta 2018; 486: 76-85

[24] Zhao DD, jiao PL, Yu J] et al. Higher Serum Uric Acid Is Associated with Higher Bone Mineral Density in Chinese Men with Type 2 Diabetes Mellitus. Int J Endocrinol 2016; 2016: 2528956

[25] Kang DH, Han L, Ouyang $X$ et al. Uric acid causes vascular smooth muscle cell proliferation by entering cells via a functional urate transporter. Am J Nephrol 2005; 25: 425-433

[26] Baldwin W, McRae S, Marek G et al. Hyperuricemia as a mediator of the proinflammatory endocrine imbalance in the adipose tissue in a murine model of the metabolic syndrome. Diabetes 2011; 60: 1258-1269

[27] Kang DH, Park SK, Lee IK, Johnson RJ. Uric acid-induced C-reactive protein expression: Implication on cell proliferation and nitric oxide production of human vascular cells. J Am Soc Nephrol 2005; 16: 3553-3562
[28] Ahn SH, Lee SH, Kim B] et al. Higher serum uric acid is associated with higher bone mass, lower bone turnover, and lower prevalence of vertebral fracture in healthy postmenopausal women. Osteoporos Int 2013; 24: 2961-2970

[29] Lean JM, Davies JT, Fuller $\mathrm{K}$ et al. A crucial role for thiol antioxidants in estrogen-deficiency bone loss. J Clin Invest 2003; 112: 915-923

[30] Han W, Bai X, Wang N et al. Association between lumbar bone mineral density and serum uric acid in postmenopausal women: A cross-sectional study of healthy Chinese population. Arch Osteoporos 2017; 12: 50

[31] Takiue Y, Hosoyamada M, Kimura M, Saito H. The effect of female hormones upon urate transport systems in the mouse kidney. Nucleosides Nucleotides Nucleic Acids 2011; 30: 113-119

[32] Shinaki M. Critical appraisal of physical rehabilitation measures after osteoporotic vertebral fracture. Osteoporos Int 2003; 14: 773-779

[33] Majumdar SR, Leslie WD, Lix LM et al. Longer duration of diabetes strongly impacts fracture risk assessment: The Manitoba BMD Cohort. J Clin Endocrinol Metab 2016; 101: 4489-4496 